\title{
Electronic Adverse Incident Reporting in Hospitals
}

\author{
Kerry Walsh, Calvin Burns*, \& Jiju Antony
}

SIOM, University of Strathclyde, Glasgow, Scotland, UK

*Strathclyde Business School, University of Strathclyde, Glasgow, Scotland, UK

\section{Corresponding Author:}

Dr. Calvin Burns

Lecturer in Organisational Behaviour

Department of Human Resource Management

Strathclyde Business School

University of Strathclyde

Graham Hills Building

50 Richmond Street

GLASGOW G1 1XU

Tel. 44 (0)141 5484251

Fax 44 (0)141 5523581

calvin.burns@strath.ac.uk 


\begin{abstract}
Purpose: The purpose of this study was to assess attitudes toward and use of an electronic adverse incident reporting system in all four hospitals in one NHS Scotland Health Board area.

Methods: A questionnaire was used to assess Medical Consultants', Managers', and Nurses' attitudes and perceptions about electronic adverse incident reporting. Actual adverse incident reporting data were also analysed.

Findings: The main findings from this study are that Consultants, Managers, and Nurses all had positive attitudes about responsibility for reporting adverse incidents. All respondents indicated that the design of and information collected by the electronic adverse incident reporting system (DATIX) was adequate but Consultants had more negative attitudes and perceptions than Managers and Nurses about DATIX. All respondents expressed negative attitudes about the amount and type of feedback they receive from reporting, and Consultants expressed more negative attitudes about how DATIX is managed than Managers and Nurses. Analysis of adverse incident reporting data found that the proportion of Consultants using DATIX to report incidents was significantly lower than that of Managers and Nurses.

Implications: The findings suggest that there are no additional barriers to incident reporting associated with the use of a bespoke electronic adverse incident reporting system as compared to other types of systems. Although an electronic adverse incident reporting system may be able to increase incident reporting and facilitate organisational learning by making it easier to report incidents and analyse incident reporting data, strong leadership within hospitals / healthcare professions (or healthcare subcultures) is still required in order to promote and sustain incident reporting to improve patient safety.
\end{abstract}

Originality: This is the first study to investigate attitudes toward and reporting behaviour on a bespoke electronic adverse incident reporting system in hospitals.

Key Words: Electronic adverse incident reporting, Patient safety, Subcultures

Paper Type: Research Paper 
In healthcare, there is growing recognition of the need to collect and analyse data on adverse incidents in order to facilitate learning and improve patient safety. The World Health Organisation (2005) has stated that an effective reporting system is the cornerstone of safe practice within a hospital or other health-care organisation. Accordingly, several countries have introduced national or system-wide reporting systems to monitor and analyse incident data (e.g. Williams \& Osborn, 2006; Spigelman \& Swan, 2005).

Reporting systems with the primary goal of quality improvement tend to be voluntary systems, and reports are usually submitted anonymously, or with confidentiality safeguards to an external agency for analysis and feedback, so that deficiencies can be addressed. However, reporting systems do not provide a reliable index of the rate of adverse incidents as there are many barriers to incident reporting (Vincent, Stanhope, \& Crowley-Murphy, 1999). In response to some of these barriers, electronic reporting systems have been introduced in many hospitals but users' views of electronic reporting systems are largely unknown. This paper shall assess the attitudes toward and use of a bespoke electronic adverse incident reporting system in all four hospitals within one NHS Scotland Health Board.

\section{Barriers to Reporting}

There are many barriers to incident reporting in healthcare. Studies have identified time constraints, cumbersome forms, lack of knowledge about how and what to report, lack of feedback, and a perceived lack of value in the reporting process as barriers to reporting (Evans, Berry, Smith, et al., 2006; Kingston, Evans, Smith et al., 2004; Lawton \& Parker, 2002; Schectman \& Plews-Ogan, 2006; Taylor, Brownstein, Christakis, et al., 2004; Waring, 2005). Also, Billings (1998) argued that fear of embarrassment, fear of punishment (of oneself and others), and fear of litigation were major reasons why healthcare workers did not report adverse incidents. Barach and Small (2000) have identified inhibitive reporting cultures and lack of adequate systems as further barriers to reporting. In the above studies, it is unclear whether the barriers to reporting that were identified were associated exclusively with traditional paper-based reporting systems. It may be the case that additional barriers to reporting may be associated with the use of bespoke electronic reporting systems.

A number of studies have also documented that doctors are less likely to report incidents and / or express favourable attitudes about incident reporting than nurses and other types of healthcare workers (Evans et al., 2006; Kingston et al., 2004; Lawton \& Parker, 2002; Taylor et al., 2004; Westbrook, Braithwaite, Travaglia, et al., 2007). This effect has also been demonstrated with respect to electronic reporting systems (e.g. Braithwaite, Westbrook, \& Travaglia, 2008). One reason for doctors' less favourable attitudes and lower rates of incident reporting may be due to the culture of medicine. Rosenthal (1999) argued that its emphasis on professional autonomy, collegiality, and self-regulation is not likely to support error reporting. Leape (2000) supported a call for a more open culture and better reporting in healthcare. Trust (between management, staff, and the public) is needed to create a cultural change in healthcare of increased incident reporting (Firth-Cozen, 2004). In a climate of trust, a reporting culture, a just culture, and a learning culture can interact to create a safety culture (Burns, Mearns, \& McGeorge, 2006) and incident reporting can then yield greater improvements in patient safety. 
It would be difficult, if not impossible, to change the reporting culture within entire healthcare systems. Allinson (2004) argued that electronic information systems and communication through information technology can be used to introduce new efficiency and services. Taylor et al. (2004) found that $45 \%$ of doctors and nurses surveyed thought an electronic format for reports would lead to increased reporting of medical errors. However, Force, Deering, Hubbe et al. (2006) suggested that as electronic reporting systems are adopted, there are likely to be increases in the numbers of detected adverse events, rather than decreases in the numbers of actual adverse events due to improvements. In order to create a safety culture, an organisation must possess a learning culture which requires it to have the willingness and the competence to draw the right conclusions from its safety information system, and the will to implement major reforms when their need is indicated (Reason, 1997).

\section{Electronic Reporting Systems}

There has been a focus in healthcare on replacing paper based reporting systems with electronic systems in order to improve delays associated with data entry and other barriers to reporting (Armondi, 2000). Many of these electronic systems are web-based (e.g. Braithwaite et al., 2008; Nakajima, Kurata, \& Takeda, 2005; Wu, Pronovost, \& Morlock, 2002) but some are on personal digital assistants or PDAs (e.g. Bent, Bolsin, Creati, et al., 2002) or have been integrated into an electronic patient record system (e.g. Haller, Myles, Stoelwinder, et al., 2004).

Heeks, Mundy and Salazar (1999) argued that health care information systems (HCIS) will fail more often than they succeed due to the mismatch between the conceptions in a system's design and the realities into which it is introduced. They proposed that the mismatch can be assessed along seven dimensions: 1) Information, 2) Technology, 3) Processes 4) Objectives and Values, 5) Staffing and Skills, 6) Management and Structures, and 7) Other Resources. They concluded that the starting point for any process of HCIS implementation must be analysis of the conceptionreality gap. Although a common barrier to reporting associated with both paper based and electronic systems appears to be lack of feedback (e.g. Braithwaite et al., 2008 identified lack of feedback as a problem in their study of a web-based system), there is a dearth of research about the implementation of bespoke electronic adverse incident reporting systems in hospitals. Walsh and Antony (2007b) called for further research to obtain the views of clinicians and managers in the potential use, modification and development of electronic adverse incident reporting in hospitals.

\section{The Current Study}

This paper compares the attitudes, and reporting behaviour of Medical Consultants, Managers, and Nurses with respect to an electronic adverse incident reporting system (DATIX) in hospitals within one NHS Scotland Health Board area. DATIX is a bespoke, commercial, integrated reporting system designed to collect information from adverse incidents and near miss events electronically. In addition to an Incident module, the system also incorporates Claims, Complaints, and Risk modules.

Prior to the current study, the Health Board had been using DATIX for two years. At the time of the study, the Health Board employed directly around 5,900 clinical staff and 3,800 non-clinical support staff across its four hospitals, which provide healthcare to a population of about 367,000 people. The purpose of the introduction of DATIX was to replace a paper system that was both 
time consuming and inefficient in raising the awareness of patient safety issues. The Executive Medical Director had expressed concern that the Health Board was unable to establish the level of adverse incidents and near misses being recorded due to the limitations of the paper system. Before the introduction of DATIX, data were collected on different databases and then recorded against a paper system. This made it difficult to ensure that the data were accurate and up to date. Thus, it was not possible for this study to compare adverse incident data reported before and after the introduction of DATIX, as the data beforehand were unavailable. For further information about DATIX and its adoption by the Health Board, see Walsh and Antony (2007a). 


\section{METHODS}

Participants. Participants were employees of an NHS Scotland Health Board. Questionnaires were sent via internal mail to a random selection of Medical Consultants, Managers, Nurses, Allied Health Professionals, and other support staff across the Health Board's four hospitals. In total, 440 questionnaires were distributed and 126 were returned (an overall response rate of 29\%); 210 were sent to Medical Consultants (response rate $=12 \%$ ), 93 to Managers (response rate $=29 \%$ ), and 70 to Nurses (response rate $=50 \%$ ). This paper shall consider the findings from respondents in the three largest occupational groups (Medical Consultants, Managers, and Nurses), which comprised $73 \%$ of respondents (seven respondents did not indicate their occupational group). The demographics of the three main occupational groups are shown in Table 1. There were no significant differences between these groups, except gender, as might be expected.

Table 1: Demographics of the sample by main occupational groups

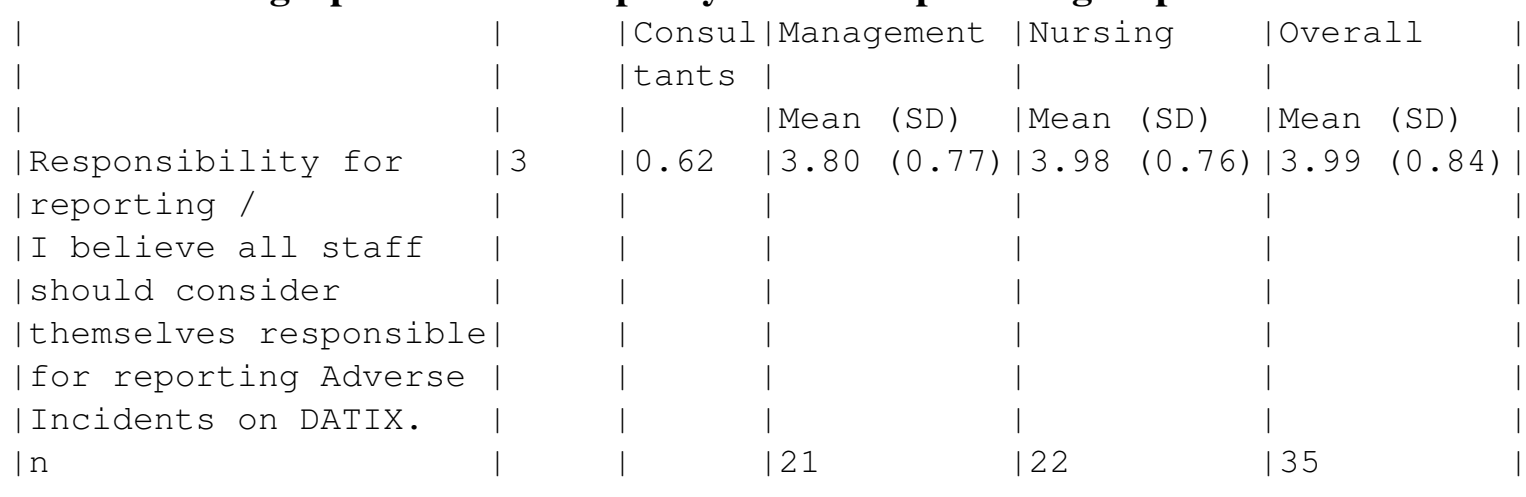

As can be seen in Table 2, somewhat positive attitudes were expressed about responsibility for reporting adverse incidents by all three occupational groups. No significant differences emerged between Consultants, Managers, and Nurses on this factor.

Design and Information. Participants were asked their views on the design of and information collected by DATIX. The four factors that emerged from the factor analysis $\mathrm{w}_{2}$ were: 1 ) Usefulness of DATIX for improving patient safety, 2) How information from DATIX informs the organisation, 3) Use of DATIX on a continuous and hospital-wide basis, and 4) Adequacy of DATIX for reporting and recording adverse incidents. The four factors extracted accounted for $63.6 \%$ of the variance and all showed reasonable internal reliability.

Table 3: Hospitals Staff's views on Design of and Information Collected by DATIX

\begin{tabular}{|c|c|c|c|c|c|c|}
\hline Factor / & I Items & Alpha & I Consultant & Manaq & ers & | Nurses \\
\hline Typical item & | & । & Is & | & & । \\
\hline & | & । & | Mean (SD) & | Mean & $(\mathrm{SD})$ & I Mean (SD) \\
\hline is of DI & 15 & 10.798 & 12.80 & 13.18 & $(0.69)$ & 13.01 \\
\hline patier & & & $(0.90)$ & | & & $(0.90)$ \\
\hline
\end{tabular}




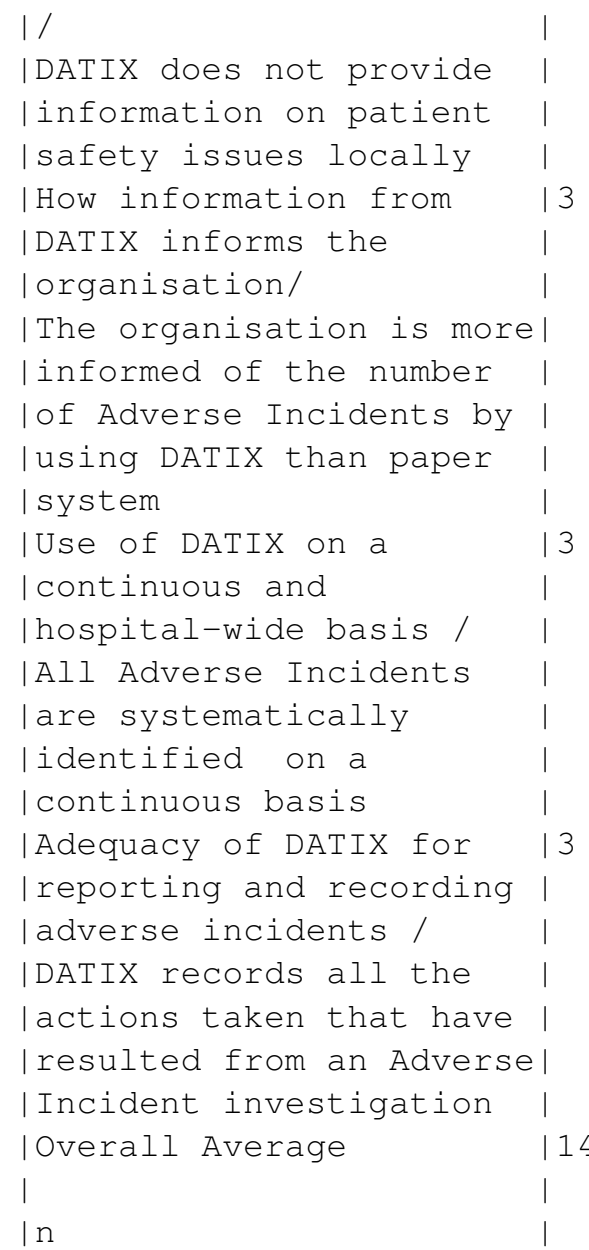

As can be seen in Table 3, mostly neutral views were expressed about the design of and information collected by DATIX. An analysis of variance (ANOVA) revealed that overall, there were significant differences between the occupational groups for the Overall Average score; $\mathrm{F}(2,77)=3.88, \mathrm{p}<0.05$. Pairwise comparisons using the Bonferroni method revealed that this was due to the fact that Consultants' views were significantly more negative than Nurses' views ( $\mathrm{p}<$ $0.05)$.

An ANOVA found that overall, there were significant differences between the occupational groups for How information from DATIX informs the organisation; $F(2,75)=3.99, p<0.05$. Pairwise comparisons using the Bonferroni method revealed that Consultants' views were significantly more negative than Nurses' views $(\mathrm{p}<0.05)$. The difference between Consultants' and Managers' views approached significance at the 0.05 level.

An ANOVA also revealed that overall there were significant differences between the occupational groups for Use of DATIX on a continuous and hospital-wide basis; $F(2,77)=9.48, p<0.001$. Pairwise comparisons using the Bonferroni method revealed that Consultants' views were significantly more negative than both Nurses' and Managers' views ( $\mathrm{p}<0.05$ for both). There were no significant differences between the occupational groups for Usefulness of DATIX for improving patient safety, and Adequacy of DATIX for reporting and recording adverse incidents.

Attitudes toward Management of DATIX. Respondents were asked their views on how management used the DATIX system. The factors that emerged from the factor analysis ${ }_{2}$ were 
Trust, Reviewing and checking use, and Feedback. The three factors extracted accounted for $73.0 \%$ of the variance and all showed reasonable internal reliability.

Table 4: Hospital Staff's Attitude towards Management of DATIX

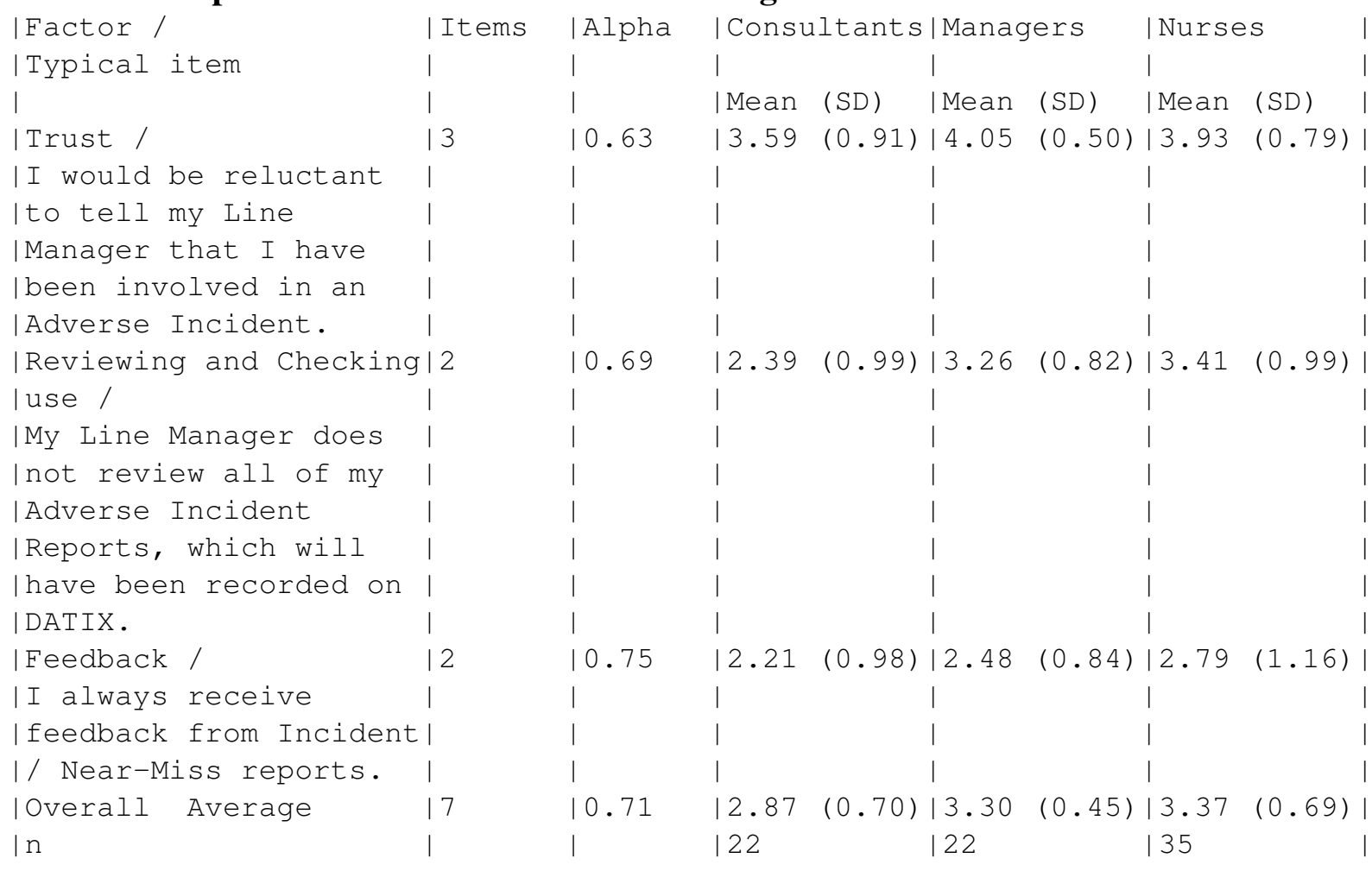

As can be seen in Table 4, all three occupational groups expressed somewhat positive attitudes about Trust, rather neutral attitudes about Reviewing and Checking Use, and negative attitudes about Feedback. The Overall Average score was neutral for all three groups.

ANOVA revealed that overall, there were significant differences between the occupational groups for the Overall Average score; $\mathrm{F}(2,76)=4.57, \mathrm{p}<0.05$. Pairwise comparisons using the Bonferroni method revealed that this was due to the fact that Consultants' views were significantly more negative than Nurses' views $(\mathrm{p}<0.05)$. The difference between Consultants' and Managers' views approached significance at the 0.05 level.

ANOVA also revealed that overall, there were significant differences between the occupational groups for Reviewing and Checking use; $\mathrm{F}(2,59)=4.19, \mathrm{p}<0.05$. Pairwise comparisons using the Bonferroni method revealed that this was due to the fact that Consultants' views were significantly more negative than Nurses' views $(\mathrm{p}<0.05)$. The difference between Consultants' and Managers' views approached significance at the 0.05 level. There were no significant differences between occupational groups for Trust, and Feedback.

Reporting Behaviour. Lastly, participants were asked "During the last year have you reported an incident or near-miss using DATIX?" As per Table 1, 56.5\% of Consultants, 47.8\% of Managers, and 71.4\% of Nurses reported that they had used DATIX to report an incident or near-miss in the past year. A chi-square test was used to investigate whether there were any significant differences in the proportion of respondents from these occupational groups using 
DATIX. The chi-square tests revealed that there were no significant differences between these occupational groups on this self-report measure of DATIX use; $\operatorname{chi}(2)=3.447, p=0.178$. This finding was surprising as nurses are more likely than doctors to complete incident reports, as noted earlier. However, in the current study, the respondents were a self-select sample (recall that the response rate for Consultants was only $12 \%$ and these Consultants may be more predisposed to incident reporting than the Consultants who did not respond to the questionnaire) and this questionnaire item may have elicited a socially desirable response.

In order to further investigate reporting trends by occupational group, data from the Health Board were examined. For the calendar year 2008 (when this survey was conducted) 47 incidents were reported on DATIX by Consultants, 3,535 incidents by Nurses, and 191 incidents by Managers. Although nurses reported the most incidents, these figures need to be considered in terms of the number of people employed in each group. In 2008, the Health Board employed 241 Consultants, 4,634 Nurses, and 161 Managers. Thus, the proportion of Consultants reporting adverse incidents was 0.195; (47 Consultant incident reports / 241 Consultants employed). The proportion of Nurses reporting adverse incidents was 0.763 . The proportion of Managers reporting adverse incidents was 1.186. While indicative of reporting trends, these proportions are somewhat crude figures. They do not take into account the possibility that an individual from an occupational group may have made more than one incident report, thereby inflating the proportion of reports made by an occupational group.

In order to test for independence between the number of incident reports and the number of people employed in each group, three chi-square tests (Managers vs. Consultants, Managers vs. Nurses, and Nurses vs. Consultants) were conducted. In each case, the test revealed that the number of incident reports was not independent of the number of people employed in each group ( $\mathrm{p}<$ 0.0001). In other words, the proportion of Managers reporting on DATIX was greater than the proportion of Consultants, and Nurses, and the proportion of Nurses reporting on DATIX was greater than the proportion of Consultants. 


\section{DISCUSSION}

The main findings from this study are that Consultants, Managers, and Nurses all had positive attitudes about responsibility for reporting adverse incidents. All respondents indicated that the design of and information collected by DATIX was adequate but Consultants had more negative attitudes and perceptions than Managers and Nurses in this respect. All respondents expressed negative attitudes about the amount and type of feedback they received from reporting, and Consultants expressed more negative attitudes about how DATIX is managed than Managers and Nurses. Analysis of adverse incident reporting data found that the proportion of Consultants using DATIX to report incidents was significantly lower than that of Managers and Nurses. These findings are consistent with those in the extant literature but importantly, they do not suggest any additional barriers to incident reporting associated with the use of a bespoke electronic adverse incident reporting system as compared to other types of systems.

The current study is not without its limitations and these should be acknowledged before further implications of this study are considered. The first limitation is that there was a small response rate from Consultants. As mentioned above, questionnaires were sent to 210 Consultants (nearly all of the Consultants employed by the Health Board) but only 25 Consultants (12\%) responded. Thus, the questionnaire data from Consultants in this study may not be representative of Consultants employed by the Health Board. It is likely that the 25 Consultants who responded to the questionnaire had more positive attitudes and perceptions about incident reporting than those who did not respond, as they were motivated to express their views by taking part in the study. Although the questionnaire data from Consultants was largely negative (apart from Responsibility for Reporting) this would suggest that the data reported gives a best case scenario for Consultants' views.

Another limitation was that this study did not compare questionnaire data between the four hospitals. Callen, Braithwaite and Westbrook (2007) noted that hospital cultures can differ which can impact on the use of technology. The Consultants and Managers in this study worked at / across all four hospitals in the Health Board but most of the Nurses tended to be based at just one of them. Due to this fact, it would have been difficult to classify many of the respondents (or for them to classify themselves) as belonging to just one of the four hospitals. However, differences in the culture of the four hospitals were not expected as they were all managed by the same senior management team (headed by the Health Board's chief executive), and the same policies, systems, and procedures were used across all four hospitals.

Lastly, this study did not compare adverse incident data reported before and after the introduction of DATIX. Doing so would have allowed for the identification of any change in the type of incidents reported and any change in the reporting rate of different occupational groups that would have presumably come from the introduction of a bespoke electronic adverse incident reporting system. However, as noted earlier, before the introduction of DATIX, data were collected on different databases and then recorded against a paper system. This made it difficult to ensure that the data were accurate and up to date. Thus, it was not possible for this study to compare adverse incident data reported before and after the introduction of DATIX, as the data beforehand were unavailable.

The implications of the current study have to do with leadership within occupational (medical, 
managerial, and nursing) subcultures with respect to adverse incident reporting in hospitals. Carroll and Quijada (2004) argued that a hospital is not a single culture but rather a fragmented collection of occupational cultures such as medicine, nursing, and management (and subcultures within, such as surgery, anaesthesiology, pharmacy, finance, and marketing). Davies, Nutley and Mannion (2000) argued that hospital subcultures may be associated with different levels of power and influence and they cite the dominance of the medical culture in the NHS and the relatively recent rise of the management culture as further evidence. Braithwaite and Westbrook (2005) noted that the culture in health care is tribal in this respect. Carroll and Quijada (2004) argued that rather than oppose existing culture, it may be more effective to build on existing cultural strengths and gradually tilt the culture, and that to do this, leaders act as role models.

Given positive attitudes about the importance of reporting, leadership within subcultures is vital in order to promote and sustain adverse incident reporting in hospitals. It requires medical directors and consultants (medical subcultures), nursing directors and nurse managers (nursing subcultures), and CEO's and non-clinical managers (managerial subcultures) to not only engage with and be seen to be engaging with the reporting system, but to ensure that their staff receive feedback when reports are made, and that information is communicated and deficiencies are addressed across the hospital. These recommendations are consistent with Evans et al.'s (2006) conclusions that to improve incident reporting, the reporting process needs to be simplified but good leadership is still needed by making it clear which incidents should be reported and by giving feedback to reporters. This should enhance perceptions of organisational trustworthiness (Gillespie \& Dietz, 2009) which should facilitate the interaction of Reason's (1997) subcomponents of a reporting culture, a just culture, and a learning culture into a safety culture.

Electronic adverse incident reporting systems may increase incident reporting by making it easier to report incidents and analyse data. However, strong leadership within hospital subcultures is still required in order to promote and sustain reporting, to facilitate organisational learning, and ultimately improve patient safety. 


\section{REFERENCES}

Allinson, C. (2004). The process of audit and control: A comparison of manual and electronic information systems. International Journal of Police Strategies and Management, 27, 183-205.

Armondi, A. (2000). Healthcare information systems: Challenges of the new millennium. Idea Group: Hershey, PA.

Bent, P.D., Bolsin, S.N., Creati, B.J., et al. (2004). Professional monitoring and critical incident reporting using personal digital assistants. Med J Aust, 177, 496-499.

Billings, C. (1998). Some hopes and concerns regarding medical event-reporting systems. Arch Pathol Lab Med, 122, 214-15.

Braithwaite, J., \& Westrbrook, M. (2005). Rethinking clinical organisational structures: An attitude survey of doctors, nurses and allied health staff in clinical directorates. $J$ Health Serv Res Policy, 10, 10-17

Braithwaite, J., Westbrook, M., \& Travaglia, J. (2008). Attitudes toward the large-scale implementation of an incident reporting system. International Journal for Quality in Health Care, 20, 184-191.

Burns, C., Mearns, K. \& McGeorge, P. (2006). Explicit and implicit trust within safety culture. Risk Analysis, 26, 1139-1150.

Callen, J.L., Braithwaite, J., \& Westbrook, J.I. (2007). Cultures in hospitals and their influence on attitudes to, and satisfaction with, the use of clinical information technology. Social Science \& Medicine, 65, 635-639.

Carroll, J.S., \& Quijada, M.A. (2004). Redirecting traditional professional values to support safety: changing organisational culture in health care. Qual. Saf. Health Care, 13, ii16-ii21.

Cronbach L.J. (1951). Coefficient alpha and the internal structure of tests. Psychometrika, 16, 297334.

Davies, H.T.O., Nutley, S.M., \& Mannion, R. (2000). Organisational culture and quality of health care. Quality in Health Care, 9, 111-119.

Evans, S.M., Berry, J.G., Smith, B.J., et al. (2006). Attitudes and barriers to incident reporting: A collaborative hospital study, Qual Saf Health Care, 15, 39-43.

Firth-Cozens, J. (2004). Organisational trust: The keystone to patient safety. Qual. Saf. Health Care, 13, 56-61.

Flin, R., Burns, C., Mearns, K., et al. (2006). Measuring safety climate in health care. Quality and Safety in Health Care, 15, 109-115. 
Force, M.V., Deering, L., Hubbe, J., et al. (2006). Effective strategies to increase reporting of medication errors in hospitals. J Nurs Adm, 36, 34-41.

Gillespie, N., \& Deitz, G. (2009). Trust repair after an organization-level failure. Academy of Management Review, 34, 127-145.

Haller, G., Myles, P.S., Stoelwinder, J., et al. (2004). Integrating incident reporting into an electronic patient record system. Journal of the American Medical Informatics Association, 14, 175-181.

Heeks, R., Mundy, D., Salazar, A. (1999). Why Health Care Information Systems Succeed or Fail. Information systems for public sector management, Working paper series, Paper no. 9. Retrieved from:

http://www.sed.manchester.ac.uk/idpm/research/publications/wp/igovernment/index.htm

Kingston, M.J., Evans, S.M., Smith, B.J., et al. (2004). Attitudes of doctors and nurses towards incident reporting: A qualitative analysis. Med J Aust, 181, 36-9.

Lawton, R. \& Parker, D. (2002). Barriers to incident reporting in a healthcare system. Qual Saf Health Care, 11, 15-18.

Nakajima, K., Kurata, Y., \& Takeda, H. (2005). A web-based incident reporting system and multidisciplinary collaborative projects for patient safety in a Japanese hospital. Qual Saf Health Care, 14, 123-129.

Reason, J. (1997). Managing the Risks of Organizational Accidents. Aldershot: Ashgate.

Rosenthal, M. (1999). How doctors think about medical mishaps. In M. Rosenthal, L. Mulcahy, \& S. Lloyd-Bostock eds. Medical mishaps. Open University Press: Buckingham.

Schectman, J.M. \& Plews-Ogan, M.L. (2006). Physician perception of hospital safety and barriers to incident reporting. Jt Comm J Qual Patient Saf, 32, 337-43.

Spigelman, A.D., \& Swan, J. (2005). Review of the Australian incident monitoring system. ANZ J Surg, 75, 657-61.

Taylor, J.A., Brownstein, D., Christakis, D.A., et al. (2004). Use of incident reports by physicians and nurses to document medical errors in pediatric patients, Pediatrics, 114, 729-735.

Vincent C., Stanhope, N., Crowley-Murphy, M. (1999). Reasons for not reporting adverse incidents: An empirical study. J Eval Clin Pract 5, 13-21.

Walsh, K. \& Antony, J. (2007a). Improving patient safety and quality: What are the challenges and gaps in introducing an integrated electronic adverse incident and recording system within health care industry? International Journal of Health Care Quality Assurance, 20, 107-115.

Walsh, K. \& Antony, J. (2007b). Quality costs and electronic adverse incident recording and 
reporting system: Is there a missing link? International Journal of Health Care Quality Assurance, 20, 307-319.

Waring, J.J. (2005). Beyond blame: Cultural barriers to medical incident reporting. Soc Sci Med, $60,1927-35$.

Westbrook, M.T., Braithwaite, J., Travaglia, J.F., et al. (2007). Promoting safety: Varied reactions of doctors, nurses and allied health professionals to a safety improvement program. Int J Health Care Qual Assur, 20, 555-571.

Williams, S.K., \& Osborn, S.S. (2006). The development of the National Reporting and Learning System in England and Wales, 2001-2005. Med J Aust, 184, 65-68S.

World Health Organisation (2005). WHO draft guidelines for adverse event reporting and learning systems: From information to action. WHO Press: Geneva.

Wu, A.W., Pronovost, P., \& Morlock, L. (2002). ICU incident reporting systems. Journal of Critical Care, 17, 86-94. 


\section{FOOTNOTES}

1. Each factor analysis used Principal Component Analysis with Varimax rotation. Principal components analysis is chosen because this approach is widely used to assess the dimensional structure of a dataset and reduce a large number of variables in to a smaller set of linear components for subsequent analyses (Dunteman, 1989; Kellow, 2006). The factor solution is rotated using varimax-rotation to improve the interpretability of the final solution.

2 The suitability of the data for factor analysis was assessed by the Kaiser-Meyer-Olkin (KMO) measure of sampling adequacy (Kaiser, 1974). The KMO measure gave a score of 0.67 for Design and Information, 0.63 for Values about Reporting, and 0.59 for Attitudes toward Management. Each of these were enough to assure the data was appropriate for factor analysis (Field, 2000; Hutcheson and Sofroniou, 1999). Eigenvalues greater than one and scree tests were used to decide on the number of factors (Kaiser, 1960, Cattell, 1978). 